\title{
A REVIEW ON MULTICOMMODITY URBAN EVACUATION PROBLEM
}

\section{Gopal Prasad Acharya and Shree Ram Khadka}

Journal of Institute of Science and Technology

Volume 21, Issue 1, August 2016

ISSN: 2469-9062 (print), 2467-9240(e)

Editors:

Prof. Dr. Kumar Sapkota

Prof. Dr. Armila Rajbhandari

Assoc. Prof. Dr. Gopi Chandra Kaphle

JIST, 21 (1), 19-27 (2016)

Published by:

Institute of Science and Technology

Tribhuvan University

Kirtipur, Kathmandu, Nepal

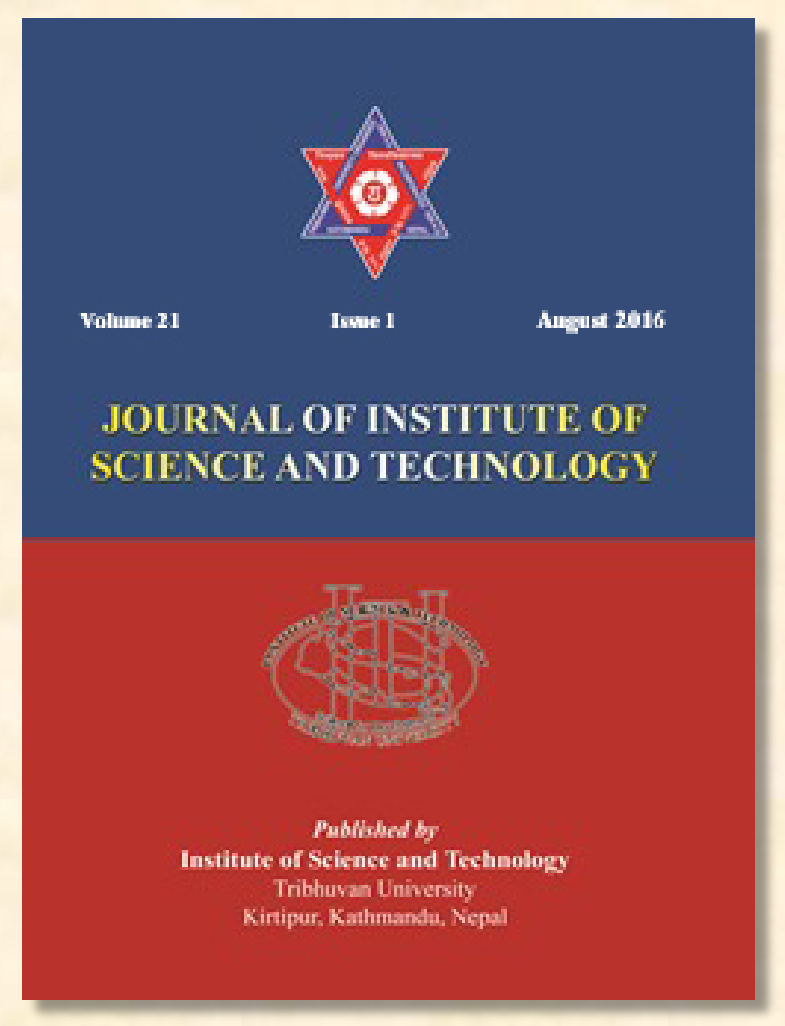




\title{
A REVIEW ON MULTICOMMODITY URBAN EVACUATION PROBLEM
}

\author{
Gopal Prasad Acharya and Shree Ram Khadka* \\ Central Department Mathematics, Tribhuvan University, Kirtipur, Nepal \\ *Corresponding email: shreeramkhadka@gmail.com
}

\begin{abstract}
Urban evacuation regards as a critical course of action that helps to re-locate the maximum number of people and property from the disaster zone of the urban area to the safe zone within the shortest possible time. Multi-commodity urban evacuation problem (MCUEP) explores and identifies the re-construction of the traffic routes to be followed in evacuation with the mobility of two or more types of the vehicles allowing two way streets. The idea of the paper is to introduce a multi-commodity urban evacuation traffic routing modeling with the bus commodity and the vehicle commodity in the street segment and intersection segment where the crossing and merging cannot be prohibited. The evacuation is made effective by sending and re-sending the buses (if necessary) and using the personal vehicles for once. The paper will give a review of MCUEP as the state of the art basically designed as a mixed integer model functioning as the remedy of the shortcomings of one-way evacuation model and put forwards a brief discussion of the solution approaches.
\end{abstract}

Key words: Emergency lanes, Multi-commodity evacuation, Evacuation planning, Disaster management

\section{INTRODUCTION}

A severe disruption of functioning of society or the community incurred due to the manmade or the natural hazard that results into the casualties of the people and loses of property is called the disaster. Natural disaster often has consequences on environmental and socio-economical system that directly damages the structures/infrastructures needed for the development and hinders the development process directly. Due to their unpredictable phenomenon, the world is suffering from the huge loss of lives and properties. The disaster management plan only can redress and regress the protective policies of such devastating disasters that help to mitigate the post disaster effects. The effective plan helps to recognize the effective traffic routes with the alternative course of action which tremendously reduces the traffic congestion along the evacuation routes. Emergency evacuation pre-planning is critical to the safe evacuation of occupants. For preplanning, efficient tools are needed to produce evacuation model that identify routes and schedules to evacuate affected population to safety in the event of natural disasters. During the emergency evacuation in urban area, all of the evacuees may not be having their own vehicles and it is not equally possible to send the buses to each and every disaster point due to the damages or other disturbances. So the people affected by the disaster are allowed to use their own vehicles in evacuation if they have and those not having own vehicles are urged to be gathered in the public spacious and less harmful places where the buses can be sent. Such gathering places may be schools, parks or any other public places and are termed as the assembly points in the succeeding part of the articles. Besides these problems, the evacuees may be in need of ambulances, emergency medicines, oxygen gas or some sorts of logistic supports. If the number of evacuees is large enough in the assembly area and cannot be picked up in a single trip by the buses, the bus vehicles should be sent to the assembly area again and again. The buses, in the starting phase, would be at the assembly point to start immediate evacuation or they may be outside the assembly area and are to be sent to the assembly zone. Similarly ambulances can be used for the medical purposes. Only the evacuee's vehicles are not sent back to the evacuation zone after their arrival to the safe zone. This establishes the need of the two way street and the use of two or more type's vehicles in disaster management. The network diagram that represents the evacuation network 
system with the arcs sets consisting slack arcs, arcs connecting the intersections to the sources or to the sinks is depicted in figure (1) and (2).

Though the movement of the vehicles in both the directions is necessary but it creates lots of conflicts in the intersection part of the road segment. If the conflicts are totally prohibited in the intersections, no feasible solution can be obtained. This is why, it is necessary to relax the crossing conflicts in the intersection. To resolve the problems, the intersection segment of the road is divided into two lanes-the public lanes and the emergency lanes. In the public lanes, all the types of vehicles i.e. the vehicles of the evacuees, bus commodities, ambulances etc. are allowed. In the emergency lanes, only the emergency vehicles are allowed. On the other hand, the street segment can be used by both the types of the vehicles. The segments are depicted below. Within the intersections, the crossing conflicts between the public lanes are completely prohibited. The crossing conflicts of the emergency lanes with the emergency one or with public lanes are relaxed. The number of emergency lanes thus created are counted and penalized. The pedestrians are not modeled directly but they are modeled with the number of the buses. For that, the numbers of pedestrians are assigned to each assembly points and the numbers of the buses are computed accordingly.

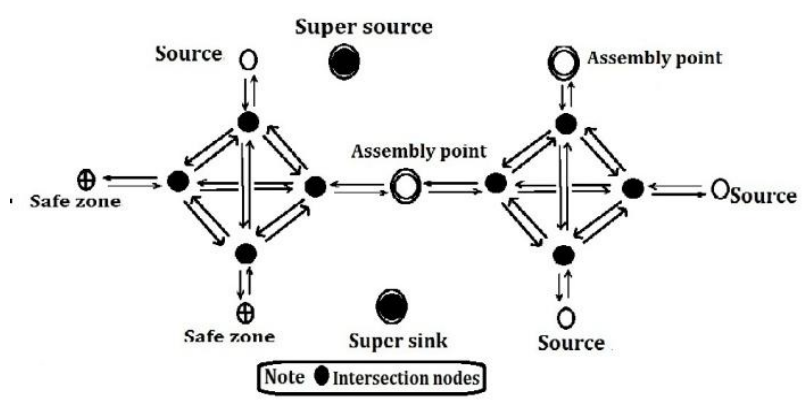

Fig (1) Sources and Sinks.

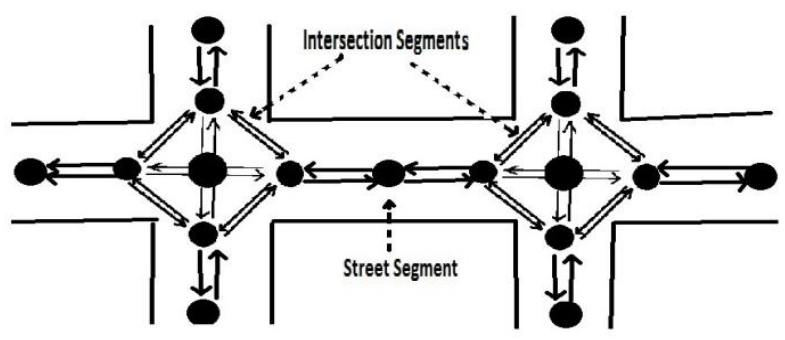

Fig (2) Street and Intersection Segment of the Road
The buses are again classified into two groups:

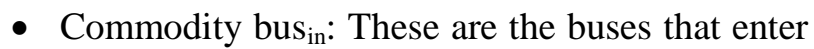
the evacuation zone from outside to pick up the evacuees from the assembly point.

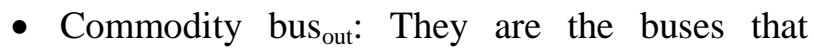
depart from the assembly zone carrying the evacuees to the safe zone outside the disaster zone. The destinations of these buses are the safe places outside the disaster area where the bus $_{\text {out }}$ sinks.

The bus $_{\text {in }}$ when reaches to the evacuation zone changes into the bus ${ }_{\text {out }}$ and departs to the safe zone. If $\Omega$ (bus $\left.{ }_{\text {out }}\right)$ and $\Omega\left(\right.$ bus $\left._{\text {in }}\right)$ representing the set of sources of bus out and bus in respectively and $\omega$ (bus $\left._{\text {in }}\right)$ and $\omega\left(\right.$ bus $\left._{\text {out }}\right)$ represent the set of the sinks of the bus in and bus out respectively, we can assume that,

$$
\begin{aligned}
& \omega\left(\text { bus }_{\text {in }}\right)=\Omega\left(\text { bus }_{\text {out }}\right) \\
& \omega\left(\text { bus }_{\text {out }}\right)=\Omega\left(\text { bus }_{\text {in }}\right)
\end{aligned}
$$

and $c o \in\left\{\right.$ bus $_{\text {in }}$, bus $\left._{\text {out }}\right\}$. Let us suppose that $\mathrm{B}_{\text {out }}$ $(\Omega)$ represents the number of buses that are estimated to leave the source which is same to the estimated number of evacuees that are assigned to the assembly points. $\mathrm{O}(\mathrm{co}, \Omega)$ is the number of buses co $\in\left\{\right.$ bus $_{\text {in }}$, bus $\left._{\text {out }}\right\}$ ready to leave the source in time $0 . \mathrm{B}_{\text {out }}(\Omega)-\mathrm{O}(\mathrm{co}, \Omega)$ is the number of buses that are still needed to transport all the evacuees to the safe zone.

A few examples of the crossing conflicts within the intersections with different number of entrances /exits are depicted in fig. no. (3).

The number of crossing on flicts within an intersection can be computed by:

$$
\alpha(\alpha-2)+\alpha \sum_{n=0}^{\alpha-3} n\{-n+(\alpha-1)\}
$$

where $\alpha \geq 3$ is the number of entrances/exits in the intersection[6]. Generally the travel time along the virtual arcs is set to zero. In the case of the bus commodity, it can be considered to the time required in the assembly or in the safe area to make the buses ready to start again. The travel time along the arcs starting from or ended at the super nodes is considered as zero and the same time is adjusted as the time required in the assembly point or at the safe zone. The time required for the pedestrians to get into the buses in the assembly area or the time required for the buses to be emptied at the safe zone or the time required for the buses to drive the evacuees to the shelter and drive back to the safe zone can be modeled with the transit time along the arcs connected to the super node. In general, the 
super source is the virtual source and the super sink is the virtual destination. Let $\mathrm{s}$ be the source point such that $s \in \Omega\left(\right.$ bus $\left._{\text {out }}\right)$. If we model the transit time $\left(b s_{\text {out }}, a \Omega\right) \geq 0$, then $\tau\left(b u s_{\text {in }}, a, \omega\right)=0$.

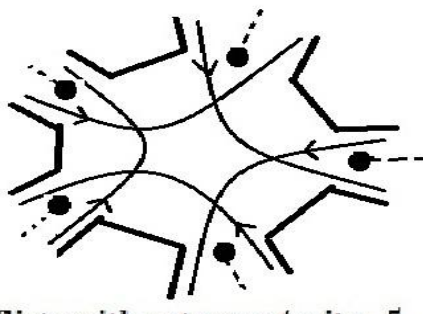

Conflicts with entrance $/$ exits $=5$ for left turn arcs
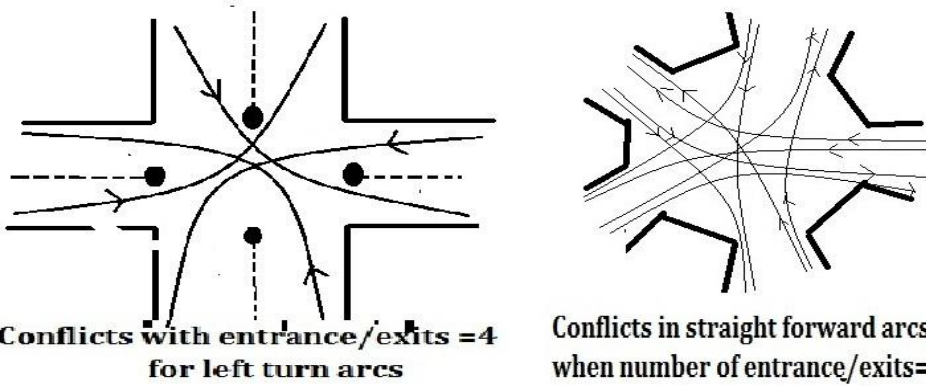

Conflicts in straight forward arcs when number of entrance/exits $=5$

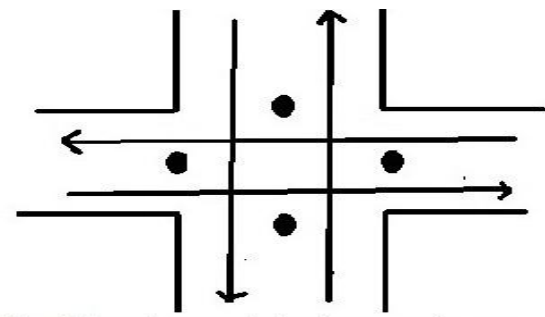

Conflicts in straight forward arcs when number of entrance/exits $=5$

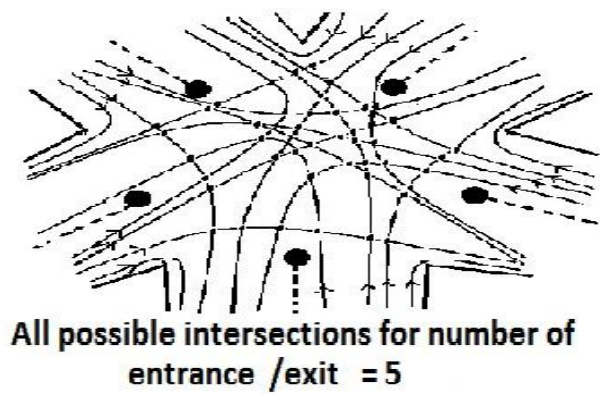

Fig.(3) Possible Conflict Points in the Intersection Segments with Different number of entrances.

\section{Present status of the problem}

Most of the evacuation models are the dynamic by nature. The transformation of the dynamic network flow problems into time-expanded form [the contribution of Ford and Fulkerson (in 1958)] has been the milestone for the development of different algorithms of quickest transshipment problem and earliest arrival flow problem for multiple sources and single sink which was indeed modified and introduced time weighted capacitated network by Wilkinson in 1971. In 2001, Hamacher and Tjandra worked together in building evacuation. Cova and Johnson introduced the concept of minimum cost flow problems with the objective of finding routes of the flow by minimizing the crossing conflicts and intersection merging in 2003. In 2006, Hamcher developed dynamic shortest path problem. A. Kimms and Sarah Bretschneider worked together in the field of pattern based urban evacuation model in 2011. In 2012, Sarah Bretschneider presented the solution of Multicommodity evacuation problem with 4 stage heuristic approach and addresses the limitations of the two stages heuristic approach and relaxation based heuristic approach. In this review article the plan is organized as follows. In section (2) the development of the multi-commodity evacuation model will be discussed and formulation of the multi-commodity urban evacuation model will be presented. In section (3) the solution approaches developed until now will be discussed. In section (4) the limitations of the model and in section (5) the concluding remarks will be given.

\section{FORMULATION OF THE PROBLEM}

Useful Notations: In the given network $G=$ $(\mathcal{N}, \mathcal{A})$ suppose that $\mathcal{N}_{\mathcal{J}}$ and $\mathcal{A}_{\mathfrak{J}}$ represent the set of intersection nodes and every possible direction (arcs) within the intersections respectively. Between every pair of neighboring intersections, a node is added and the arcs connecting the source or sink node to the intersection node is denoted by $\mathcal{A}_{\mathcal{C}}$. Let $\mathcal{C}=\left\{b_{\text {bus }}\right.$, bus $_{\text {out }}$, veh $\}$ be the set of the commodities and $\mathcal{C}_{e}=\left\{\right.$ bus $\left._{\text {in }}, b u s_{\text {out }}\right\}$ be the set of emergency vehicles. The other notations are mentioned below:

$\mathcal{N}: \mathcal{N}=\cup_{c o \in \mathcal{C}} \Omega(c o) \cup \cup_{c o \in \mathcal{C}} \omega(c o) \cup \mathcal{N}_{\mathcal{J}} \cup\{\omega \cup \Omega)$ is the set of nodes under the consideration such that $\Omega\left(\right.$ bus $\left._{\text {out }}\right)=\omega\left(\right.$ bus $\left._{\text {in }}\right), \omega\left(\right.$ bus $\left._{\text {out }}\right)=\Omega\left(\right.$ bus $\left._{\text {in }}\right)$, $\mathcal{A}_{\mathcal{J}}^{\mathcal{L}}$ denotes the set of all the left turn $\operatorname{arcs}, \mathcal{A}_{\mathcal{J}}^{S F}$ is the set of the straight forward arcs and $\mathcal{A}_{\mathcal{J}}^{R}$ represents the set of the right turn arcs. $\mathcal{A}_{C}$ 
denotes the set of arcs connecting intersections with sources and sink, $\mathcal{A}_{\Omega}(\mathrm{CO})$ is the set of the arcs pointing from the super source $\Omega$ to sources of the commodity co $\in \mathcal{C}$. Similarly $\mathcal{A}_{\omega}(\mathrm{Co})$ is the set of arcs emerging from the sinks $c o \in \mathcal{C}$. Combining we get $\mathcal{A}=\mathcal{A}_{\mathcal{J}} \cup \mathcal{A}_{\mathcal{C}} \cup \cup_{C o \in \mathcal{C}} \mathcal{A}_{\Omega}(c o) \cup$ $\mathrm{U}_{c o \in \mathcal{C}} \mathcal{A}_{\omega}(\mathrm{co})$.

\section{Parameters:}

- $\mathcal{T}=\{1,2,3,4, \ldots, \mathrm{T}\}$ is set of time points discretized. ; $\tau(\operatorname{co}, \mathrm{i}, \mathrm{j})=$ travel time from node $\mathrm{i}$ to node $\mathrm{j}$ on the $\operatorname{arcs}(\mathrm{i} ; \mathrm{j}) \in \mathcal{A}$ of commodity $c o \in \mathcal{C} . \mathcal{B S}(s) \subset \mathcal{T}$ is the possible schedule for the commodity $c o \in \mathcal{C}_{e}$ in source $s \in$ $\Omega(\mathrm{co}) ; O(\mathrm{co}, \mathrm{s})$ is the number of commodity co $\in \mathcal{C}$ in the source $s \in \Omega(\mathrm{co})$ at the time $\mathrm{t}=$ 0 and $B_{\text {out }}(s)$ is the number of buses bus out that have to leave the assembly point $s \in$ $\Omega$ (bus out $)$ to rescue waiting there. Also $c^{i n}(i, j)$ and $c(i, j)$ denote the inflow and the total flow of the $\operatorname{arc}(i, j) \in \mathcal{A}_{\mathcal{J}} \cup \mathcal{A}_{\mathcal{C}}$ per lane and per time unit respectively.

- $\mathcal{L}(\mathrm{i}, \mathrm{j})=$ the number of available lanes on the $\operatorname{arcs}(i, j) \in \mathcal{A}_{\mathfrak{j}} \cup \mathcal{A}_{\mathcal{C}}$ and $O(c o, s)=$ the number of the commodity co $\in \mathcal{C}$ in the source $s \in \Omega(c o)$ at the time $\mathrm{t}=0$ :
- $\psi^{\text {in }}(\mathrm{co}) / \psi_{j}^{\text {in }}(\mathrm{Co})=$ consumption of the inflow capacity of the inflow of the commodity $c o \in \mathcal{C}$ outside and within the intersections per time period; $\frac{\psi(c o)}{\psi_{J}(c o)}=$ consumption of the total street capacity per unit time of oneunit of flow capacity $c o \in \mathcal{C}$ on the arcs outside/within the intersection.

- $\mathcal{B S}(s) \subset \mathcal{T}=$ possible bus schedule for the commodity co $\in \mathcal{C}$ in source $s \in \Omega$ (co).

- $\mathrm{x}(\mathrm{co} ; \mathrm{i} ; \mathrm{j} ; \mathrm{t}) \geq 0=$ inow of the commodity co $\in \mathcal{C}$ on $\operatorname{arcs}(i, j) \in \mathcal{A}$ that leaves node $\mathrm{i}$ at point in time $t \in \mathcal{T}$ and enters node $\mathrm{j}$ at point in time $t+\tau(c o, i, j)$ with $\mathrm{x}(\mathrm{co} ; \mathrm{i} ; \mathrm{j} ; \mathrm{t})=0 \forall$ co $\in \mathcal{C} ;(i, j) \in \mathcal{A} \quad t \in\{-\tau(i, j), \ldots .,-1\}$ for the ease ofnotation.

- $l_{e}(i, j) \in \mathbb{N}_{0}=$ Number of emergency lanes assigned to the $\operatorname{arcs}(i, j) \in \mathcal{A}_{J}$ with $l_{e}(i, j)=$ $0 \forall(i, j) \in \mathcal{A}_{J}^{R}$ and $l_{p}(i, j) \in \mathbb{N}_{0}$ represents number of the public lanes assigned to $\operatorname{arc}(i, j) \in \mathcal{A}_{\mathfrak{J}} \cup \mathcal{A}_{\mathcal{C}}$

- $y_{g}(i, j) \in\{0,1\}=$ binary variable with $y_{g}(i, j)=1$ if it is allowed to assign apositive number of public lanes and emergency lanes to direct $(i, j) \in \mathcal{A}_{\mathcal{J}}^{\mathcal{L}} \cup \mathcal{A}_{\mathcal{J}}^{S F}$ or if not $(=0) ; n_{e}(q) \geq$ $0=$ number of emergency lanes within intersections $q \in \mathcal{J}$ with $\alpha_{q} \geq 2$.

The objective functions: This model concerns maximizing the evacuees in the shortest possible time by finding the appropriate traffic routing in the street network with intersections.

$$
\sum_{(c o \in \mathcal{C})} \sum_{d \in \omega(c o)} \sum_{t \in \mathcal{T}} P r_{c o} . t . x(c o, d, \omega, t)+\sum_{i \in \mathcal{J} ; \alpha_{i \geq 2}} P_{i} . n_{e}(i) .
$$

This is the weighted sum of the priority cost and the weighted number of the emergency lanes. Constrains are given as follows:

- Flow conversion constraints for the commodities $b_{u s}$, $, b u s_{\text {out }}, v e h$ : The flow cannot be stable at any node at time $t$ except in the super nodes. The total inflow of a node in time period $t$ is equal to the total outflow in time period $t$.

$$
\sum_{j \in \mathcal{N},(j, i) \in \mathcal{A}} \mathrm{x}(\mathrm{co}, \mathrm{j}, \mathrm{i}, \mathrm{t}-\mathrm{r}(\mathrm{co}, \mathrm{j}, \mathrm{i}))=\sum_{j \in \mathcal{N},(j, i) \in \mathcal{A}} \mathrm{x}(\mathrm{co}, \mathrm{i}, \mathrm{j}, \mathrm{t}) \forall \operatorname{co} \in \mathcal{C} ; \mathrm{i} \in \mathcal{N}-\{\Omega, \omega\} ; \mathrm{t} \in \mathcal{T} .
$$

Avoidance of irregular behaviors within the intersections (type I): The flow entered from one intersection node is to be traversed to exactly one and only one of the next intersection nodes and there cannot a node in between two nodes through which the flow enters within the intersection and passes out from it.

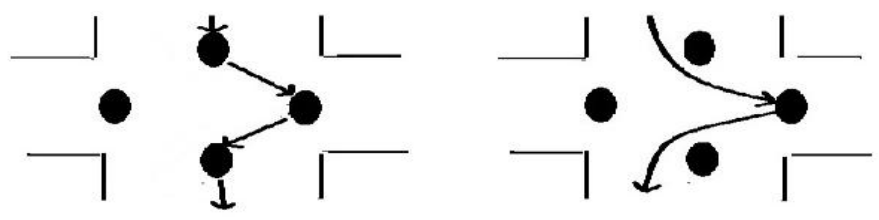

Fig.(4) Irregular behavior I 


$$
\sum_{k \in \mathcal{N}_{J}:(k, i) \in \mathcal{A}} \mathrm{x}(\mathrm{co}, \mathrm{k}, \mathrm{i}, \mathrm{t}-\mathrm{r}(\mathrm{k}, \mathrm{i}))=\mathrm{x}(\mathrm{co}, \mathrm{i}, \mathrm{j}, \mathrm{t}) \quad \forall \operatorname{co} \in \mathrm{C}, \mathrm{i} \in \mathrm{N} ;(\mathrm{i}, \mathrm{j}) \in \mathcal{A}_{C} ; t \in \mathcal{T}
$$

- Avoidance of irregular behavior of type II: The flow entering from a node of the intersection and passing through the next node of the intersection cannot turn back from the street segment to enter to the same intersection i.e. none of the evacuees is allowed to enter the street and turn directly to opposite side of the original flow.
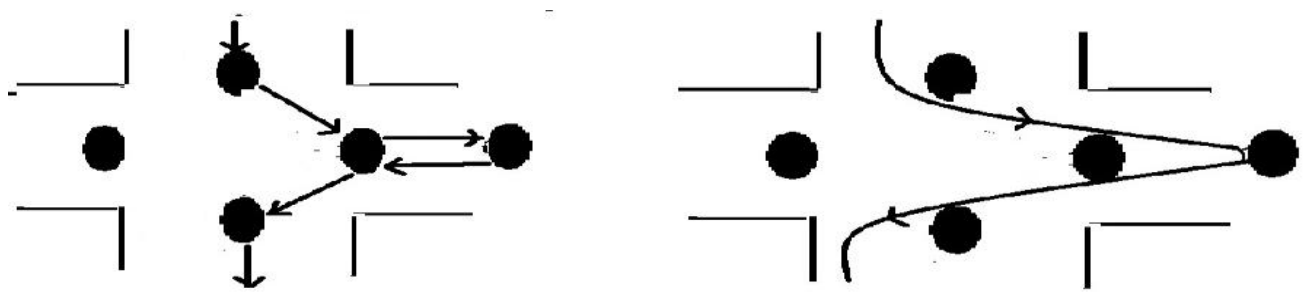

Fig (5) Irregular Behavior II

$$
\mathrm{x}(\mathrm{co}, \mathrm{i}, \mathrm{j}, \mathrm{t}-\tau(\mathrm{i}, \mathrm{j})) \leq \sum_{(j, k) \in \mathcal{A}_{C} \cup A_{j} \cup A_{D}(c o)-\{j, i\}} x(c o, j, k, t) \forall \operatorname{co} \in \mathcal{C} ;(\mathrm{i}, \mathrm{j}) \in \mathcal{A}_{C} ; t \in \mathcal{T} \text {. }
$$

- Vehicles of Evacuees and Buses constraints: All vehicles of evacuees used in evacuation must leave the source points within the time horizon $\mathcal{T}=\{0,1,2, \ldots \mathrm{T}\}$.

and

$$
\sum_{t \in \tau} \mathrm{x}(\text { veh, } \Omega, \mathrm{s}, \mathrm{t})=(\text { veh, } \mathrm{s}) \forall \mathrm{s} \in \Omega(\text { veh }) \text {. }
$$

- Every vehicle carrying evacuees from the evacuation zone must enter the safe zone within the time horizon $\mathcal{T}=\{1,2,3, \ldots \ldots, \mathrm{T}\}$

$$
\sum_{d \in \omega(v e h)} \sum_{t \in \mathcal{T}} x(v e h, \omega, t)=\sum_{s \in \Omega(v e h)} O(v e h, s) .
$$

- Number of Buses Constraints: The buses not used under the prescribed schedule are not taken into account. A possible schedule of buses bus in $_{\text {and }}$ and bus $_{\text {out }}$ that starts at the sources $\Omega$ (bus (bin $_{\text {in }}$ ) and $\Omega$ (bus $_{\text {out }}$ ) respectively is given as:

$$
\begin{aligned}
& x(c o, \Omega, s, t) \leq 1 \forall c o \in \mathcal{C}, s \in \Omega(c o) ; t \in \mathcal{B} \mathcal{S}(s) \\
& x(c o, \Omega, s, t)=0 \forall c o \in \mathcal{C}, s \in \Omega(c o) ; t \in \mathcal{T}-\mathcal{B} \mathcal{S}(s) .
\end{aligned}
$$

- The number of buses that have to leave the assembly point $s \in \Omega\left(\right.$ bus $\left._{\text {out }}\right)$ is $\mathrm{B}_{\text {out }}(\mathrm{s})$ used to transport all the pedestrians to safe zone is:

$$
\sum_{t \in \mathcal{T}} x\left(\text { bus }_{\text {out }}, \Omega, s, t\right)=B_{\text {out }}(s) \quad \forall s \in \Omega\left(\text { bus }_{\text {out }}\right) .
$$

- All buses that are needed to transport the pedestrians to the safe zone have to leave the evacuation zone within the time horizon is :

$$
\sum_{d \in \omega\left(b u s_{\text {out }}\right)} \sum_{t \in \mathcal{T}} x\left(\text { bus }_{\text {out }}, d, \omega, t-\tau\left(\text { bus }_{\text {out }}, d, \omega\right)\right)=\sum_{s \in \Omega\left(\text { bus }_{\text {out }}\right)} B_{\text {out }}(\Omega) .
$$

Here the time $\tau\left(b_{\text {out }}, d, \omega\right)$ is the time needed to empty the bus or the time needed for the bus to drive the evacuees to the shelter. It shows that the total number of buses that are sent from the evacuation is same as the total number of buses that reach to the destination. 
- Capacity of number of buses bus out at assembly point $\alpha \in \Omega\left(b u s_{\text {out }}\right)$ : The capacity of the number of the buses going out from the assembly point should not exceed to the capacity of the buses at the assembly point in the beginning minus the capacity of the buses that have gone out from the assembly point until time $T-1$ plus the capacity of the number of buses entering to the assembly point until time $T-1$.

$$
\begin{aligned}
& \mathrm{x}\left(\text { bus }_{\text {out }}, \Omega, \alpha, \mathrm{t}\right) \leq \mathrm{O}\left(\text { bus }_{\text {out }}, \alpha\right)+\sum_{\lambda=0}^{t-r\left(\text { bus }_{\text {in }}, \alpha, \omega\right)-1} x\left(b u s_{\text {in }}, \alpha, \omega, \lambda\right) \\
& -\sum_{\lambda=0}^{t-1} x\left(\text { bus }_{\text {out }}, \Omega, \alpha, \lambda\right) \forall \alpha \Omega\left(\text { bus }_{\text {out }}\right) ; \mathrm{t} \in T \forall \alpha \Omega\left(\text { bus }_{\text {out }}\right) ; \mathrm{t} \in \mathcal{T} .
\end{aligned}
$$

An assumption is that $\omega\left(\right.$ bus $\left._{\text {in }}\right)=\Omega\left(\right.$ bus $\left._{\text {out }}\right)$ but if not then the following constraints would be used.

$$
\mathrm{x}\left(\text { bus }_{\text {out }}, \Omega, \alpha, \mathrm{t}\right) \leq \mathrm{O}\left(\text { bus }_{\text {out }}, \alpha\right)-\sum_{\lambda=0}^{t-1} x\left(\text { bus }_{\text {out }}, \Omega, \alpha, \lambda\right) \forall \alpha \Omega\left(b_{\text {bus }}\right) ; \mathrm{t} \in \tau .
$$

- Capacity of the number of buses bus in $_{\text {at }}$ afe area $\alpha \in \Omega\left(\right.$ bus $\left._{\text {in }}\right)$ :

$$
\begin{aligned}
\mathrm{x}\left(\text { bus }_{\text {in }}, \Omega, \alpha, \mathrm{t}\right) & \\
& \leq \mathrm{O}\left(\mathrm{bus}_{\text {in }}, \alpha\right)+\sum_{\lambda=0}^{t-r\left(\text { bus }_{\text {out }}, \alpha, \omega\right)-1} x\left(b u s_{\text {out }}, \alpha, \omega, \lambda\right) \\
& -\sum_{\lambda=0}^{t-1} x\left(\text { bus }_{i n}, \Omega, \alpha, \lambda\right) \quad \forall \alpha \Omega\left(b u s_{\text {in }}\right) ; \mathrm{t} \in \mathcal{T} .
\end{aligned}
$$

- Similarly the capacity of the number of the buses at the safe point in destination at time $t$ does not exceed to the capacity of the number of the buses that are there in the beginning minus the capacity of the number of the buses that have left the point until time T-1 plus the capacity of the number of buses that arrived there until t- $\tau\left(\right.$ bus $\left._{\text {out }}, \alpha, \omega\right)-1$.

If $\alpha \notin \Omega\left(\right.$ bus $\left._{\text {out }}\right)$ :

$$
\mathrm{x}\left(b_{i n}, \Omega, \alpha, \mathrm{t}\right) \leq O\left(b u s_{i n}, \alpha\right)-\sum_{\lambda=0}^{t-1} x\left(b u s_{i n}, \Omega, \alpha, \lambda\right) \forall a \in\left(b u s_{i n}\right) ; \mathrm{t} \in \mathcal{T} .
$$

- Traffic Routing Constraints: Emergency lanes are created only in the intersections and not in the street segment. In the street segment only the public lanes are considered and in the intersection both of them are taken:

The number of assigned lanes in the street segment must not exceed the available lanes:

$$
\ell_{p}(i, j)+\ell_{p}(j, i) \leq \mathcal{L}(i, j) \forall(i, j) \in \mathcal{A}_{\mathcal{C}} ; j \in \mathcal{N}_{\mathcal{J}} .
$$

And the number of public and emergency lanes in the intersections must not exceed to the number of available lanes.

$$
\ell_{p}(i, j)+\ell_{p}(j, i)+\ell_{e}(i, j)+\ell_{e}(j, i) \leq \mathcal{L}(i, j) \forall(i, j) \in \mathcal{A}_{j} ; j \in \mathcal{N}_{\mathfrak{J}} .
$$

- Coupling Constraints: If the follow along an arc within an intersection is allowed, the variable is assigned value 1 which makes possible for the assignment of the positive number of lanes along that direction. Right turn arcs are not considered since they do not lead to crossing conflicts.

$$
\ell_{g}(i, j) \leq \mathcal{L}(\mathrm{i}, \mathrm{j}) y_{g}(\mathrm{i}, \mathrm{j}) \forall(\mathrm{i}, \mathrm{j}) \in A_{J}^{L} \cup A_{J}^{S F}: g \in\{p, e\} .
$$

- Prohibition of crossing conflict: Within the intersection, no crossing conflicts of two left arcs are allowed. Similarly the crossings of the straight forwards arcs are also prohibited.

$$
y_{p}(i, j)+y_{p}(k, h) \leq 1 \forall(\mathrm{i}, \mathrm{j}),(\mathrm{k}, \mathrm{h}) \in A_{J}^{L} \cup A_{J}^{S F} .
$$


- Counting emergency Lanes: The crossing conflicts of emergency lanes with the emergency lanes or with the public lanes are permitted but the emergency lanes are counted and penalized in the objective function.

$$
\sum_{(i, j) \in A_{j}(q)} y_{e(i, j) \leq n_{e}(q) \forall q \in J: \alpha_{q} \geq 2}
$$

- The merging of lanes is restricted:

$$
\begin{gathered}
\sum_{(k, i) \in A^{*}(\delta . i)}\left(l_{e}(k, i)+l_{p}(k, i)\right) \leq l_{p}(i, j)+(\delta-1) \\
\forall q \in J ; i \in N_{j}(q) ;(i, j) \in A_{C} ; \delta \in\left\{1,2, \ldots \ldots ., \delta_{j}-1\right\} ; \\
A *_{(\delta, i)} \in\left\{A \subseteq A_{J}(q)|A|=\delta, A \ni(k, i) \text { for ak } \in N_{J}(q)\right\}
\end{gathered}
$$

$\psi(\mathrm{co}) / \psi_{j}(\mathrm{co})$ and $\psi_{j}(\mathrm{co}) / \psi_{j}^{\text {in }}(\mathrm{co})$ denotes the consumption of the total capacity and inflow capacity on arcs between/within intersections of one unit of flow of commodity co $\in \mathcal{C}=\left(\right.$ bus $_{\text {in }}$, bus $_{\text {out }}$, veh $)$ per unit time respectively.
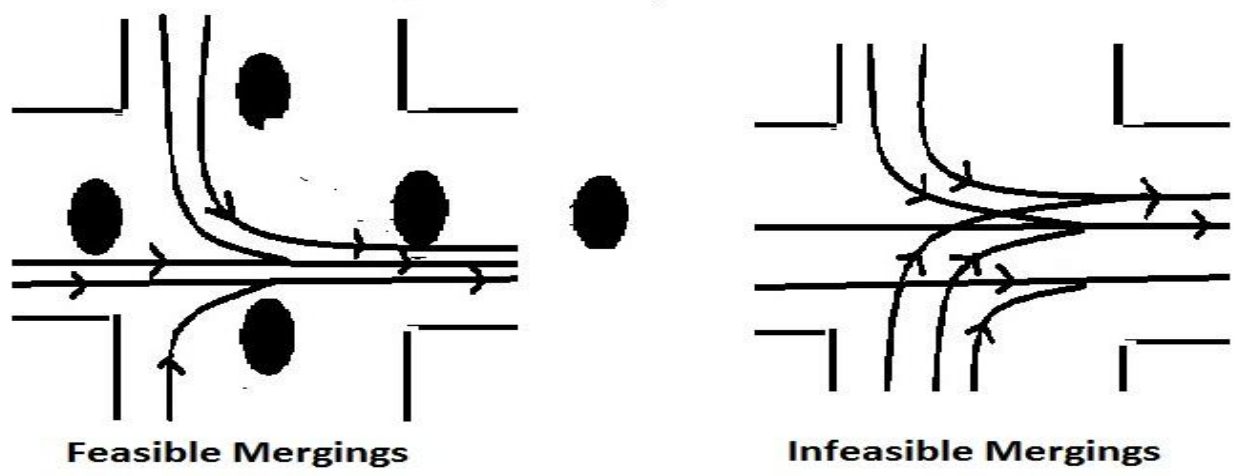

Infeasible Mergings

Fig (6) Feasible and Infeasible Merging's.

- Inflow capacity constraints: The inflow is restricted within intersection for buses and vehicles.

$$
\sum_{c o \in C} \psi_{j}^{i n}(c o) x(c o, i, j, t) \leq c_{i n}(i, j)\left(l_{e}(i, j)+l_{p}(i, j)\right) \quad \forall(i, j) \in A_{j}: t \in \mathcal{T} .
$$

- Vehicles are only allowed to use public lanes. The inflow capacity is to be restricted for the vehicles depending on the number of public lanes $1 p$

$$
\psi_{j}^{\text {in }}(\text { veh }) x(v e h, i, j, t) \leq c^{i n}(i, j) l_{p}(i, j) \quad \forall(i, j) \in A_{j} ; t \in \mathcal{T}
$$

Again,

$$
\sum_{c o \in C} \psi_{j}^{i n}(c o) x(c o, i, j, t) \leq c o^{i n}(i, j) l_{p}(i, j) \quad \forall(i, j) \in A_{C} ; t \in \mathcal{T} .
$$

- Total street capacity constraints: The total street capacity is restricted on intersection $\operatorname{arcs} \mathcal{T}_{\mathrm{J}}$ for buses and vehicles:

$$
\sum_{c o \in C} \sum_{\lambda=t-r(c o, i, j)+1} \psi_{J}(c o) x(c o, i, j, \lambda) \leq c(i, j)\left(l_{e}(i, j)\right)+l_{p}(i, j) \forall(i, j) \in A_{J} ; t \in \mathcal{T} .
$$

- Vehicles of evacuees are restricted to use only public lane within intersection

$$
\sum_{\lambda=t-\tau(c o, i, j)+1} \psi_{J}(v e h) x(v e h, i, j, \lambda) \leq c(i, j)\left(l_{p}(i, j)\right) \quad \forall(i, j) \in A_{J} ; t \in \mathcal{T} .
$$

- The total street capacity associated to the connecting arcs $\mathcal{A}_{\mathrm{C}}$ is permitted to be shared by buses and vehicles 


$$
\sum_{c o \in C} \sum_{\lambda=t-\tau(c o, i, j)+1} \psi_{J}(c o) x(c o, i, j, \lambda) \leq c(i, j)\left(l_{p}(i, j)\right) \quad \forall(i, j) \in A_{C} ; t \in \mathcal{T}
$$

So far the commodity is concerned, different travel times for the different commodities will be chosen and one of the priority option first in first out can be applied. It ultimately allows the possibility of overtaking which is mapped with the travel time.

\section{SOLUTION PROCEDURE}

Analytic solution of the problem has not been developed yet. The problem consists of the multicommodity means of transportation that flow on two way streets. Due to these complexities, more numbers of constraints are necessarily developed and the problem is oriented to the heuristic solution approach that consist of the different aggregation level of the street network. But there is no proof of the problem to be NP hard. The complete solution of the problem is obtained with the Four-staged Heuristic Approach. The first two stages help to compute the flow quantity on the network and the traffic routing on the reduced network which can be utilized to construct the total network. Stage 3 deals with the construction of the sub-graphs for every single intersection and the fourth stage helps to resume the composite network consisting of the sub-graphs constructed in the third step.

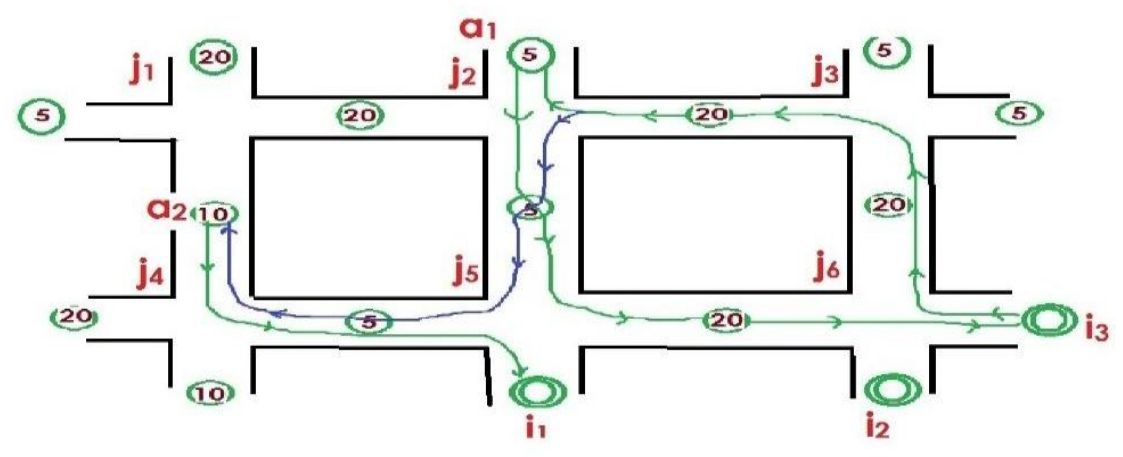

Fig.7: The Route of the Bus Commodity in an Optimal Solution

In the first stage, the flow of the emergency vehicles with corresponding constraints is considered and the number of lanes for the emergency flow for every street segment is determined in the reduced network. For the emergency vehicles at most one lane per street section is assigned. Then in the second stage, the vehicles of the evacuees are included. The flow of all the commodities is computed under the corresponding constraints and the number of lanes for the evacuation vehicles has to be determined. In the third stage, every intersection is considered separately and intersection mode incorporating the result obtained in stage 2 and it is solved on every single sub graph associated with an intersection. The constraints of the intersections like the prohibition of the crossing conflicts or the restricting of the merging of lanes are taken into account. Lastly the number of lanes of connecting $\operatorname{arcs} \mathrm{A}_{\mathrm{C}}$ and intersection arcs $\mathrm{A}_{\mathrm{J}}$ generated with step 2 and step 3 are fixed in the multi-commodity evacuation model and the inflow of all the commodities and the objective function values are computed. The solution of one of the problem by using SIPLEX-10 in a computer installed with AMPL is shown in fig (7).

\section{LIMITATION OF THE MODEL}

The two components of the objective function are the weighted sum of the flows entering the sink and the weighted number of emergency lanes that are to be minimized separately. The two components indirectly rely on each other. It is because, if high penalty cost is penalized as compared to the priority cost, it may lead to the route much longer to reach to the sink. If the penalty cost becomes less, it indirectly brings rushes in the intersection and traffic routing becomes unclear within the intersection because there will be a large number of emergency lanes. This is why a better understanding is required while setting the two components of the objective functions. Since the 
different priorities for the different commodities can be offered, there is chance to overtake the vehicle with less priority by the vehicle with greater priority. In such a case, the first come first out priority level may not be functioning properly. Another issue is that in a narrow street segment, the shorter travel time commodity may not be able to overtake the commodity with the longer travel time. In the model, the computation would be very much difficult if the different travel times and different priorities to the commodities are allocated. Since the crossing conflicts are relaxed, there may be the hostile meeting of the buses with the vehicles. Due to these reasons, the conflict management becomes a great challenge to the expert. These are the few cases which cannot be prohibited in this model. So the major limitation of the multi-commodity model is that the simultaneous time of crossing of the bus and the vehicles cannot be prohibited. The evacuees should be informed about the traffic routing in time and a trained personnel is needed to enforce and implement the changed traffic routing. There may be the practical computational problems due to the lack of memory or a long time factor.

\section{CONCLUSION}

We have discussed the mixed integer evacuation problem with the Multi-commodity means of transportation allowing them in the two way street. The crossing conflicts of emergency-emergency vehicles and emergency-public vehicles within the intersections are relaxed whereas other conflicts are discarded and feasible merging conflicts are accepted to get the optimal number of evacuees and the traffic routing of the corresponding solution is found by heuristic approach. A new type of the lane is introduced in the intersection named as emergency lane the weighted number of emergency lanes is termed as the penalty cost and we focused to minimize it. Starting from the stage 1 up to stage 3 a feasible traffic routing is generated and with the help of which the flows of the evacuees, the commodities and objective functional values are computed. Since only two types of the commodities are under study, it can be extended to more than two commodities in evacuation as a future work.
The uncertainties may be directly incorporated in the model that has the direct or indirect effect in the evacuation. Additionally, the effect on the traffic routing can be the new field of study that can be performed by extending the model by adding the stochastic events.

\section{ACKNOWLEDGMENTS}

The research of Shree Ram Khadka was supported by the European Commission in the framework of Erasmus Mundus and within the project cLINK and Kantipur Engineering College.

\section{REFERENCES}

Aeronson J. E. 1989: A Survey of Dynamic Network Flows. Annals of operation research, 20:1-66.

Bretschneider S. and Kimms. 2011: A basic Mathematical Model for Evacuation Problems in Urban Areas Transportation research part A, 45:523-539.

Bretschneider S. and Kimms. 2012: Pattern-based Evacuation Planning for Urban Areas. European Jornal of operation research, 216124.

Bretschneider S. 2012: Mathematical Models for Evacuation Planning in Urban Areas. A lecturer notes in economics and mathematical system. Springer-Verlag Berlin Heidelberg, 145-161.

Ford L. R., Fulkerson.1974: Flows in Networks, The Rand Corporation Princeton University Press, Princeton New Jersey, 2-22.

Hall A., Hippler and Skutella M. 2003. Multicommodity flows over time, efficient algorithms and complexity. Lecture Notes in Computer Science Springer, Berlin. 2719:397-409.

Osman M. S. and Balaram 2012: Application of static uncapacitated multi-commodity flow models to dynamic capacity constraints evacuation path networks. Proceedings of international conference on industrial engineering, 1718-1720. 